Research Paper

International Journal of Medical Sciences

ISSN 1449-1907 www.medsci.org 2008 5(4):224-229

(C) Ivyspring International Publisher. All rights reserved

\title{
Significant association between Helicobacter pylori infection and serum C-reactive protein
}

\author{
Yoshiko Ishida ${ }^{1}, K_{\text {Koji Suzuki², Kentaro Taki }}^{3}$, Toshimitsu Niwa ${ }^{3}$, Shozo Kurotsuchi ${ }^{4}$, Hisao Ando 5 , Akira \\ Iwase ${ }^{6}$, Kazuko Nishio', Kenji Wakai', Yoshinori Ito', Nobuyuki Hamajima ${ }^{1}$
}

1. Department of Preventive Medicine/Biostatistics and Medical Decision Making, Nagoya University Graduate School of Medicine, Nagoya, Japan

2. Department of Public Health, Fujita Health University School of Health Sciences

3. Department of Clinical Preventive Medicine, Nagoya University Graduate School of Medicine, Nagoya, Japan

4. Department of Obstetrics and Gynecology, Toyota Kosei Hospital, Toyota, Japan

5. Center for Reproductive Medicine, Toyohashi Municipal Hospital, Toyohashi, Japan.

6. Department of Obstetrics and Gynecology and Department of Maternal and Perinatal Medicine, Nagoya University Graduate School of Medicine, Nagoya, Japan.

Correspondence to: Ms. Yoshiko Ishida, Department of Preventive Medicine / Biostatistics and Medical Decision Making, Nagoya University Graduate School of Medicine, 65 Tsurumai-cho, Showa-ku, Nagoya 466-8550 Japan, TEL:+81-52-744-2132, FAX:+81-52-744-2971, e-mail: mitton@med.nagoya-u.ac.jp

Received: 2008.05.09; Accepted: 2008.07.23; Published: 2008.07.24

Background: Helicobacter pylori (H. pylori) infection in gastric mucosa may cause systemic inflammatory reaction. This study aimed to examine the association between the infection and serum high sensitivity C-reactive protein (hsCRP).

Methods: Subjects were comprised of three groups; 453 health checkup examinees from Yakumo town inhabitants in Hokkaido, Japan (YTI, 153 males and 300 females), 449 health checkup examinees (ENUH, 273 males and 176 females), and 255 female patients of an infertility clinic (PIC), Nagoya University Hospital. Twenty participants with hsCRP more than $1 \mathrm{mg} / \mathrm{dl}$ were excluded from the analysis. Those with hsCRP more than $0.1 \mathrm{mg} / \mathrm{dl}$ were defined as high hsCRP individuals. $H$. pylori infection status was examined with a serum IgG antibody test.

Results: When the three groups were combined, the geometric mean of hsCRP concentration was significantly higher among the seropositives $(0.047 \mathrm{mg} / \mathrm{dl})$ than among the seronegatives $(0.035 \mathrm{mg} / \mathrm{dl}) ; \mathrm{p}<0.0001$ by a $t$-test. The percentage of high hsCRP individuals was also higher in the seropositives than in the seronegatives among any group; $23.3 \%$ and $20.1 \%$ in YTI, $22.0 \%$ and $16.0 \%$ in ENUH, and $32.7 \%$ and $18.7 \%$ in PIC, respectively, although the difference was significant only in ENUH. The summary odds ratio of the high hsCRP for the seropositives relative to the seronegatives was 1.38 (95\% confidence interval, 1.01-1.89), when age, sex, body mass index, smoking, and subject group were adjusted by a logistic model.

Conclusions: In three groups, hsCRP was higher among the infected individuals. The summary odd ratio indicated that $H$. pylori infection could influence the serum hsCRP level.

Key words: C-reactive protein, Helicobacter pylori infection, inflammation, serum antibody, smoking

\section{Introduction}

Helicobacter pylori (H. pylori) is a spiral-shaped, gram-negative bacterium that inhabits the stomach mucosa in more than $50 \%$ of Japanese adults, and further more from child to elderly in developing countries [1]. This bacterium can elicit lifelong inflammatory and immune responses with release of various bacterial and host-dependent cytotoxic substances [2], resulting in chronic gastritis, peptic ulcer, and gastric cancer [3].
Epidemiological studies have suggested that $H$. pylori infection might be involved in the pathogenesis of coronary heart disease [4-6]. The suspicion about $H$. pylori involvement in the pathological lesions is based on the following: (i) local inflammation can have systemic effects; (ii) H. pylori gastric infection is a chronic process that lasts for decades; and (iii) persistent infection induces chronic inflammatory and immune responses that can induce lesions both local and remote sites from the primary infection site $[7,8]$. The 
manifestation may be caused by potential mechanisms with direct or indirect actions [9]. The direct effects on the vascular wall could include endothelial injury and dysfunction through circulating endotoxins, smooth muscle proliferation, and local inflammation. The indirect effects are more often pronounced, including elevation of inflammatory mediators with proinflammatory, procoagulant, and atherogenic action, production of cross-reactive antibodies, as well as nutrient/vitamin malabsorption and metabolic disturbances such as overproduction of ammonia by the bacterium.

C-reactive protein (CRP) is an acute-phase reactant that originates from the liver. CRP has many clinical and biological effects and can be used for the diagnosis and follow-up of various inflammatory and traumatic processes [10]. There is strong evidence that CRP is a powerful predictor of incident cardiovascular events independent of levels of LDL cholesterol, all levels of the Framingham risk score, and the metabolic syndrome [11-19]. The basal CRP level in individuals free from acute illness is reproducible [20]. Its determinants reportedly include age and smoking [21], while substantial heritability (35-40\%) was also reported in familial aggregation studies [22].

Examining the association between $H$. pylori infection and serum CRP levels seemed important to elucidate the relevance of $H$. pylori infection with coronary heart disease, especially atherosclerosis. The previous studies on the association, however, provided inconsistent results [23, 24]. This study aimed to examine the association in three different Japanese groups.

\section{Subjects and methods}

\section{Study subjects}

The first group (YTI) was 524 apparently healthy inhabitants, who attended a health check-up at Yakumo town, Hokkaido, Japan in 2006. Among them, 505 agreed to participate in the study with written informed consent. Lifestyle data collected with a self-administered questionnaire, as well as residual blood samples, were available from 459 subjects. Six subjects with serum high-sensitivity CRP (hsCRP) $>1$ $\mathrm{mg} / \mathrm{dl}$ were excluded from the analysis, because they might have an inflammatory disease. Remaining 453 subjects (153 males and 300 females) aged from 39 to 90 years were used for the present study.

The second group (ENUH) incorporated health checkup examinees at Department of Clinical Preventive Medicine, Nagoya University Hospital, who visited the department between June 2003 and February 2004. After the explanation of the study, 476 agreed lifestyle questionnaire and donation of $7 \mathrm{ml}$ peripheral blood for research purposes. Among them, 453 subjects could determine the presence of $H$. pylori infection and measure the hsCRP. Those with hsCRP more than $1 \mathrm{mg} / \mathrm{dl}$ were also excluded, remaining 449 subjects (273 males and 176 females) aged from 17 to 89 years for the present study.

The third group (PIC) consisted of 264 female patients at an infertility clinic, who underwent treatment for infertility at Department of Obstetrics and Gynecology or Department of Maternal and Perinatal Medicine, Nagoya University Hospital between February 2001 and December 2004. Stored blood samples of the patients were available for research purposes. Those with hsCRP more than $1 \mathrm{mg} / \mathrm{dl}$ were similarly excluded, remaining 255 females aged from 21 to 48 years. All the three studies were approved by the Ethics Committee of Nagoya University School of Medicine (approval number 398, 52, 278 for YTI, ENUH and PIC, respectively).

Determination of serum H. pylori infection and hsCRP value

Sera were separated from blood samples immediately after blood draw. They were kept in a deep freezer at $-80{ }^{\circ} \mathrm{C}$ until analyses. A commercially available direct ELISA kit ("E Plate 'Eiken' H. pylori Antibody" from Eiken Kagaku, Tokyo, Japan) was used to determine the presence of $H$. pylori infection. The seropositive was defined as $H$. pylori IgG antibody more than $10 \mathrm{U} / \mathrm{ml}$. The hsCRP was measured with a particle-enhanced immunoturbidometric latex agglutination assay by SRL Co. Ltd., Tokyo. The assay could detect $0.004 \mathrm{mg} / \mathrm{dl}$ of CRP. Undetectable CRP values were recorded as $0.002 \mathrm{mg} / \mathrm{dl}$. The staff in the laboratory was blinded to the seropositivity of $H$. pylori antibody.

\section{Statistical analysis}

The difference in geometric mean of serum hsCRP between $H$. pylori positive subjects and negative subjects was statistically tested with a $t$-test, because $\log _{\mathrm{e}}$-transformed hsCRP distributed symmetrically with one peak. Participants with hsCRP more than 0.1 $\mathrm{mg} / \mathrm{dl}$ were defined as high hsCRP individuals in this study. The proportion of high hsCRP subjects was compared between the seropositives and the seronegatives with a Fisher's exact test. Odds ratios (ORs) and $95 \%$ confidence intervals (CIs) of the seropositives compared with the seronegatives for high hsCRP were calculated using unconditional logistic regression models, adjusting for age $(<30,30-39,40-49,50-59$, $60-69$, or $\geqq 70$ years), sex, body mass index (BMI) $(<25$, or $\geqq 25 \mathrm{~kg} / \mathrm{m}^{2}$ ), smoking (never, former, current, or unknown), and study group. The heterogeneity in the OR among study groups was tested with the $Q$ statis- 
tic. The data were analyzed by the statistical software "STATA/SE version 9.0" (College Station, TX, USA).

\section{Results}

The sex, age, hsCRP level, BMI, and smoking habits of the participants according to the study group are summarized in Table 1 . In YTI, females were dominant $(66.2 \%)$, and $65.3 \%$ were participants aged 60 years or older. In ENUH, males were dominant $(60.8 \%)$, and age was distributed over a wider range. PIC was the group of only females aged under 50 years, because they were sampled from patients of an infertility clinic. The hsCRP distributed from undetectable level close to $1 \mathrm{mg} / \mathrm{dl}$ in any group, and the frequency of high hsCRP individuals was comparable among the three groups. The prevalence of $H$. pylori infection in YTI was 62.1\% (95/153) in males and 54.7\% $(164 / 300)$ in females, 39.9\% (109/273) and 36.9\% (65/176) in ENUH, respectively, and 20.4\% (52/255) in PIC. In total, the prevalence was $47.9 \%(204 / 426)$ in males and $38.4 \%(281 / 731)$ in females.

Table 1 Characteristics of subjects from three study groups

\begin{tabular}{|c|c|c|c|c|}
\hline Characteristics & $\begin{array}{c}\text { YTI* }^{*} \\
(n=453)\end{array}$ & $\begin{array}{l}\text { ENUH }^{\dagger} \\
(n=449)\end{array}$ & $\begin{array}{c}\text { PIC } \\
(n=255)\end{array}$ & $\begin{array}{c}\text { Total } \\
(n=1,157)\end{array}$ \\
\hline \multicolumn{5}{|l|}{ Sex } \\
\hline Males & $153(33.8)$ & $273(60.8)$ & $0(0.0)$ & $426(36.8)$ \\
\hline Females & $300(66.2)$ & $176(39.2)$ & $255(100.0)$ & 731 (63.2) \\
\hline \multicolumn{5}{|l|}{ Age (years) } \\
\hline $19-29$ & $0(0.0)$ & $19(4.2)$ & $53(20.8)$ & $72(6.2)$ \\
\hline $30-39$ & $5(1.1)$ & 97 (21.6) & 177 (69.4) & $279(24.1)$ \\
\hline $40-49$ & $34(7.5)$ & $111(24.7)$ & $25(9.8)$ & $170(14.7)$ \\
\hline $50-59$ & $118(26.0)$ & $116(25.8)$ & $0(0.0)$ & $234(20.2)$ \\
\hline $60-69$ & $171(37.7)$ & $80(17.8)$ & $0(0.0)$ & 251 (21.7) \\
\hline$\geqq 70$ & 125 (27.6) & $26(5.8)$ & $0(0.0)$ & 151 (13.1) \\
\hline \multicolumn{5}{|l|}{ BMI $\left(\mathrm{kg} / \mathrm{m}^{2}\right)$} \\
\hline$<25$ & $296(65.3)$ & 358 (79.7) & $0(0.0)$ & 654 (56.5) \\
\hline$\geqq 25$ & 157 (34.7) & $91(20.3)$ & $0(0.0)$ & $248(21.4)$ \\
\hline Unknown & $0(0.0)$ & $0(0.0)$ & $255(100.0)$ & $255(22.1)$ \\
\hline \multicolumn{5}{|l|}{ hsCRP (mg/dl) } \\
\hline$\leqq 0.1$ & $354(78.1)$ & $370(82.4)$ & $200(78.4)$ & 924 (79.9) \\
\hline$>0.1$ & 99 (21.9) & 79 (17.6) & 55 (21.6) & $233(20.1)$ \\
\hline Geometric mean & 0.047 & 0.038 & 0.038 & 0.040 \\
\hline 1st-3rd quartile & $0.022-0.090$ & $0.019-0.072$ & $0.011-0.076$ & $0.018-0.082$ \\
\hline Range & $0.002-0.973$ & $0.002-0.923$ & $0.002-0.994$ & $0.002-0.994$ \\
\hline \multicolumn{5}{|c|}{ (1) } \\
\hline Never & $280(61.8)$ & $230(51.2)$ & $0(0.0)$ & $510(56.7)$ \\
\hline Former & 97 (21.4) & $126(28.1)$ & $0(0.0)$ & $223(24.8)$ \\
\hline Current & $76(16.8)$ & $91(20.3)$ & $0(0.0)$ & 167 (18.6) \\
\hline Unknown & $0(0.0)$ & $2(0.5)$ & $255(100.0)$ & $257(22.2)$ \\
\hline
\end{tabular}

YTI*: Yakumo Town Inhabitants, ENUH't: Examinees of Nagoya University Hospital, PICł: Patients of Infertility Clinic, Nagoya University Hospital.

Percentages are shown in parentheses.
Table 2 shows associations of categorized hsCRP ( $\leqq 0.1 \mathrm{mg} / \mathrm{dl}$ or $>0.1 \mathrm{mg} / \mathrm{dl}$ ) with background factors. In YTI, ENUH and whole participants, no significant difference was found in the prevalence of hsCRP between sexes, although the high hsCRP was more prevalent in males of YTI and in females of ENUH. There was no significant difference in the hsCRP level across age groups. The geometric mean of hsCRP level was $0.051 \mathrm{mg} / \mathrm{dl}$ for $H$. pylori positive subjects and $0.042 \mathrm{mg} / \mathrm{dl}$ for H. pylori negative subjects in YTI, 0.043 $\mathrm{mg} / \mathrm{dl}$ and $0.036 \mathrm{mg} / \mathrm{dl}$ in ENUH, $0.045 \mathrm{mg} / \mathrm{dl}$ and $0.028 \mathrm{mg} / \mathrm{dl}$ in PIC, respectively. Corresponding 1st-3rd quartile was 0.025-0.094 and 0.020-0.082 in YTI, 0.023-0.072 and 0.018-0.071 in ENUH, and 0.016-0.143 and 0.010-0.061 in PIC. In total, the geometric mean of hsCRP level was $0.047 \mathrm{mg} / \mathrm{dl}$ for $H$. pylori positive subjects and $0.035 \mathrm{mg} / \mathrm{dl}$ for $H$. pylori negative subjects, with the 1st-3rd quartile of 0.023-0.093 and 0.015-0.073, respectively. The hsCRP geometric mean was significantly higher in subjects with $H$. pylori positive subjects than in those without ( $\mathrm{p}<0.0001$ by a $t$-test).

In PIC, the high hsCRP level was observed more frequently among subjects with $H$. pylori infection than among those without it, with a statistically significant difference; $32.7 \%$ vs. $18.7 \%$, $p=0.037$ (Table 3). A similar difference in the prevalence of high hsCRP level was also detected in the pooled population $(23.5 \%$ vs. $17.7 \%, \mathrm{p}=0.017)$. In YTI and ENUH groups, the proportion of high hsCRP level was similarly higher in those with $H$. pylori infection compared with those without it, but the difference was not significant; $\mathrm{p}=0.491$ for $\mathrm{YTI}$ and $\mathrm{p}=0.127$ for $\mathrm{ENUH}$.

Table 3 also shows OR and 95\% CI of H. pylori seropositivity for the high hsCRP. The ORs were 1.18 (95\% CI, 0.74-1.90) in TYI, 1.37 (95\% CI, 0.81-2.31) in ENUH, and 2.20 (95\% CI, 1.11-4.37) in PIC, after adjustment for age, sex, BMI, and smoking habits. The $\mathrm{Q}$ test for heterogeneity showed the differences in the OR among the three groups was not significant ( $\mathrm{p}=0.337)$. The summary OR was 1.38 (95\% CI, 1.01-1.89), with allowance for study groups; 1.20 (95\% CI, 0.73-1.95) in males and 1.61 (95\% CI, 1.09-1.38) in females. Because the median of the age in pooled data was 52, we divided the data into two groups at the age; the younger group (age $\leqq 52$ years) and the older group (age $>52$ years). The summary OR of the younger group adjusted for sex, BMI, smoking, and subject groups was 1.60 (95\% CI, 1.00-2.55), while the corresponding OR of the older group was 1.27 (95\% CI, 0.85-1.90). 
Table 2 Associations between the serum level of hsCRP and background factors

\begin{tabular}{|c|c|c|c|c|c|c|c|c|}
\hline \multirow[b]{2}{*}{ Characteristics } & \multicolumn{2}{|c|}{$\begin{array}{c}\mathrm{YTI}^{*} \\
(\mathrm{n}=453)\end{array}$} & \multicolumn{2}{|c|}{$\begin{array}{l}\text { ENUH }{ }^{\dagger} \\
(n=449)\end{array}$} & \multicolumn{2}{|c|}{$\begin{array}{c}\text { PIC } \ddagger \\
(n=255)\end{array}$} & \multicolumn{2}{|c|}{$\begin{array}{c}\text { Total } \\
(n=1,157)\end{array}$} \\
\hline & Low $\$$ & High" & Low $\$$ & High" & Low $\$$ & High" & Low $\$$ & High" \\
\hline \multicolumn{9}{|l|}{ Sex } \\
\hline Males & $111(72.5)$ & $42(27.5)$ & $228(83.5)$ & $45(16.5)$ & $0(0.0)$ & $0(0.0)$ & $339(79.6)$ & $87(20.4)$ \\
\hline Females & $243(81.0)$ & $57(19.0)$ & $142(80.7)$ & $34(19.3)$ & $200(78.4)$ & 55 (21.6) & $585(80.0)$ & $146(20.0)$ \\
\hline p-value & \multicolumn{2}{|c|}{0.054} & \multicolumn{2}{|c|}{0.449} & \multicolumn{2}{|l|}{-} & \multicolumn{2}{|c|}{0.879} \\
\hline \multicolumn{9}{|l|}{ Age (year) } \\
\hline $19-29$ & $0(0.0)$ & $0(0.0)$ & 17 (89.5) & $2(10.5)$ & 39 (73.6) & $14(26.4)$ & $56(77.8)$ & $16(22.2)$ \\
\hline $30-39$ & $5(100.0)$ & $0(0.0)$ & $82(84.5)$ & $15(15.3)$ & $140(79.1)$ & $37(20.9)$ & $227(81.4)$ & 52 (18.6) \\
\hline $40-49$ & $29(85.3)$ & $5(14.7)$ & $94(84.7)$ & $17(15.3)$ & $21(84.0)$ & $4(16.0)$ & $144(84.7)$ & $26(15.3)$ \\
\hline $50-59$ & $97(82.2)$ & $21(17.8)$ & $93(80.2)$ & $23(19.8)$ & $0(0.0)$ & $0(0.0)$ & $190(81.2)$ & $44(18.8)$ \\
\hline $60-69$ & $130(76.0)$ & $41(24.0)$ & $63(78.8)$ & $17(21.2)$ & $0(0.0)$ & $0(0.0)$ & $193(76.9)$ & $58(23.1)$ \\
\hline$\geqq 70$ & $93(74.4)$ & $32(25.6)$ & $21(80.8)$ & $5(19.2)$ & $0(0.0)$ & $0(0.0)$ & $114(75.5)$ & $37(24.5)$ \\
\hline p-value & \multicolumn{2}{|c|}{0.371} & \multicolumn{2}{|c|}{0.793} & \multicolumn{2}{|c|}{0.575} & \multicolumn{2}{|c|}{0.255} \\
\hline \multicolumn{9}{|l|}{ BMI (kg/m²) } \\
\hline$<25$ & $249(84.1)$ & 47 (15.9) & $302(84.4)$ & 56 (15.6) & $0(0.0)$ & $0(0.0)$ & $551(84.3)$ & $103(15.8)$ \\
\hline$\geqq 25$ & $105(66.9)$ & $52(33.1)$ & $68(74.7)$ & $23(25.3)$ & $0(0.0)$ & $0(0.0)$ & $173(69.8)$ & 75 (30.2) \\
\hline Unknown & $0(0.0)$ & $0(0.0)$ & $0(0.0)$ & $0(0.0)$ & $200(78.4)$ & 55 (21.6) & $200(78.4)$ & 55 (21.6) \\
\hline p-value & \multicolumn{2}{|c|}{$<0.001$} & \multicolumn{2}{|c|}{0.044} & \multicolumn{2}{|l|}{-} & \multicolumn{2}{|c|}{$<0.001$} \\
\hline \multicolumn{9}{|l|}{ Smoking } \\
\hline Never & $225(80.4)$ & 55 (19.6) & $190(82.6)$ & $40(17.4)$ & $0(0.0)$ & $0(0.0)$ & $415(81.4)$ & 95 (18.6) \\
\hline Former & 73 (75.3) & $24(24.7)$ & $103(81.8)$ & $23(18.3)$ & $0(0.0)$ & $0(0.0)$ & $176(78.9)$ & 47 (21.1) \\
\hline Current & $56(73.7)$ & $20(26.3)$ & 75 (82.4) & $16(17.6)$ & $0(0.0)$ & $0(0.0)$ & $131(78.4)$ & 36 (21.6) \\
\hline Unknown & $0(0.0)$ & $0(0.0)$ & $2(100.0)$ & $0(0.0)$ & $200(78.4)$ & 55 (21.6) & $202(78.6)$ & $55(21.4)$ \\
\hline p-value & \multicolumn{2}{|c|}{0.324} & \multicolumn{2}{|c|}{0.990} & - & & \multicolumn{2}{|c|}{0.589} \\
\hline Total & $354(78.1)$ & $99(21.9)$ & $370(82.4)$ & 79 (17.6) & $200(78.4)$ & 55 (21.6) & $924(79.8)$ & $233(20.1)$ \\
\hline
\end{tabular}

YTI*: Yakumo Town Inhabitants, ENUH': Examinees of Nagoya University Hospital, PICł: Patient of Infertility Clinic, Nagoya Unversity Hospital, Low ${ }^{\S}:$ hsCRP $\leqq 0.1 \mathrm{mg} / \mathrm{dl}$, High" $:$ hsCRP $>0.1 \mathrm{mg} / \mathrm{dl}$, and p-value": Fisher's exact test.

Percentages are shown in parentheses.

Table 3 Associations of the serum level of hsCRP with $H$. pylori seropositivity and odds ratio (OR) and $95 \%$ confidence interval (CI) of H. pylori for hsCRP

\begin{tabular}{|c|c|c|c|c|c|c|}
\hline & H. pylori & Low $^{*}$ & High $^{\dagger}$ & OR $(95 \%$ CI) & $\mathrm{aOR}^{\ddagger}(95 \% \mathrm{CI})$ & $\mathrm{aOR}^{\S}(95 \% \mathrm{CI})$ \\
\hline YTI" $^{\|}$ & Seronegative & $155(79.9)$ & $39(20.1)$ & 1 [Reference] & 1 [Reference] & 1 [Reference] \\
\hline$n=453$ & Seropositive & 199 (76.8) & $60(23.2)$ & $1.20[0.76-1.89]$ & $1.13[0.71-1.79]$ & $1.18[0.74-1.90]$ \\
\hline ENUH $^{\pi}$ & Seronegative & $220(84.0)$ & $42(16.0)$ & 1 [Reference] & 1 [Reference] & 1 [Reference] \\
\hline $\mathrm{n}=449$ & Seropositive & $131(78.0)$ & $37(22.0)$ & $1.49[0.92-2.44]$ & $1.38[0.83-2.32]$ & $1.37[0.81-2.31]$ \\
\hline PIC $^{* *}$ & Seronegative & 165 (81.4) & 38 (18.7) & 1 [Reference] & 1 [Reference] & 1 [Reference] \\
\hline$n=255$ & Seropositive & 35 (67.3) & 17 (32.7) & 2.11 [ $1.07-4.16]$ & $2.20[1.11-4.37]$ & \\
\hline Pooled $^{\dagger \dagger}$ & Seronegative & $553(82.3)$ & $119(17.7)$ & 1 [Reference] & 1 [Reference] & 1 [Reference] \\
\hline $\mathrm{n}=1,157$ & Seropositive & $371(76.5)$ & $114(23.5)$ & 1.45 [ 1.07-1.97] & $1.37[1.01-1.86]$ & 1.38 [ 1.01-1.89] \\
\hline
\end{tabular}

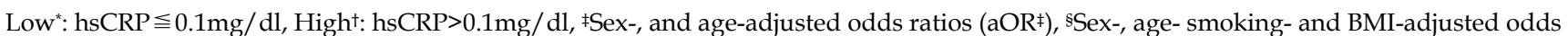
ratios (aORs), YTI" : Yakumo Town Inhabitants, ENUHף: Examinees of Nagoya University Hospital, PIC**: Patients of Infertility Clinic, Nagoya University Hospital, Pooledtt: three studies were combined, and the ORs were adjusted additionally for the study groups using dummy variables.

Percentages are shown in parentheses.

\section{Discussion}

The present paper examined the association of $H$. pylori seropositivity with hsCRP in the range of 0.002 to $1.0 \mathrm{mg} / \mathrm{dl}$, based on three different Japanese groups with 1,157 subjects in total. Although the background of the three groups was different, the sex, age, BMI, and smoking adjusted OR of the seropositive for hsCRP $>0.1 \mathrm{mg} / \mathrm{dl}$ relative to $\mathrm{hsCRP} \leqq 0.1 \mathrm{mg} / \mathrm{dl}$ was larger than unity in all three groups. The summary OR was 1.38 (95\% CI, 1.01-1.89), indicating that
H. pylori seropositivity was associated with a higher hsCRP among those with hsCRP $\leqq 1 \mathrm{mg} / \mathrm{dl}$. We tested the heterogeneity of the OR for high CRP levels in relation to the seropositivity among three groups. Because no significant differences in the OR were found among study groups, we pooled the data derived from three different populations.

Although H. pylori infection is associated with coronary artery disease, the pathway to elevate the disease risk is not clear. There are several possibilities for the mechanism underlying a causal role of $H$. pylori 
infection in endothelial dysfunction. First, H. pylori may have the direct effect on the structure and function of vascular endothelial cells. Extract of H. pylori has been reported to induce a disturbance of proliferation and apoptosis and to decrease viability of cultured vascular endothelial cells [25]. The second possibility is the nutritional effect of $H$. pylori [26]. An infection from $H$. pylori may cause malabsorption of folate, vitamin B6, and vitamin B12. This nutritional defect could lead to failure of methylation by 5-methyl-tetrahydrofolic acid and subsequent hyperhomocysteinanemia, which is toxic to endothelial cells. In our past study, serum folate was examined as a molecule connecting H. pylori infection with systemic diseases, resulting in no association between the seropositivity and serum folate [27]. CRP is also one of the candidate molecules, but there were limited reports on the association with $H$. pylori infection.

A study on the effects of $H$. pylori eradication among 78 patients in Turkey reported that serum CRP was significantly reduced among 57 participants with successful eradication, but not among 21 participants in whom the eradication failed [23]. Another study reported that increasing age, smoking, symptoms of chronic bronchitis, H. pylori and Chlamydia pneumoniae infections, and BMI were all associated with raised concentrations of CRP [24]. The present study demonstrated that $H$. pylori infection may slightly elevate serum CRP, thereby may increase systemic disease risk.

CRP is an easily measurable substance in blood. Increased levels of this protein indicate acute inflammation. Many studies have shown that high levels of CRP also indicate an increased risk of suffering from a heart attack or stroke. The CRP test is not specific, and the levels fluctuate daily and throughout the day. The levels increase as a result of numerous factors including aging, alcohol use, smoking, eating a high protein diet, coffee consumption, low levels of physical activity, chronic fatigue, high blood pressure, BMI, high triglycerides, rheumatoid arthritis, rheumatic fever, insulin resistance and diabetes [28], as well as heart attack. Medicines containing estrogen (oral contraceptive and hormone replacement therapy) may also elevate CRP levels. In this study, we took factors, sex, age, BMI, and smoking into account, although information on BMI and smoking was not available for PIC group. Among the female subjects, smokers and fatty ladies were relatively rare.

The present study has several limitations. First, $H$. pylori infection was determined by only serum antibody, not by the other diagnosis methods with a higher sensitivity and specificity, such as a urease breath test. Since the present analysis was based on stored blood samples from multi-purpose studies, alternative methods could not be applied for the detection of H. pylori infection. Second, the information on the other factors that affect serum CRP level was not available. For example, chronic infections other than $H$. pylori could confound the association with $H$. pylori infection, since it seemed plausible that $H$. pylori infected individuals might be vulnerable to other infections which elevate CRP. The adjustments of the potential confounding factors might not be enough to confirm the association with $H$. pylori infection. Third, this study was a cross-sectional study so that the causal inference was not possible directly. Finally, the effects of medication on CRP were not removed. Since several drugs, such as statins, were reported to influence CRP levels [29, 30], the adjustment for such medications could allow clear interpretation on the observed association.

If $H$. pylori has effects on the function of vascular endothelial cells, serum CRP could be one of the molecules to connect both. H. pylori infection stimulates the production of proinflammatory cytokines such as tumor necrosis factor (TNF), interleukin (IL) -6, and IL-8 [31, 32]. H. pylori was detected not only in gastric mucosa but also in human atherosclerotic plaque $[33,34]$, and the expression of intercellular adhension molecule- 1 was higher in plaques containing H. pylori than in those without it [33]. The production of CRP is regulated by cytokines, principally interleukins $[35,36]$, whose effects are modified by other cytokines and growth factors [37], as well as by hormones such as cortisol and insulin [38]. Production of cytokines and stress hormones may be altered in conditions other than inflammation or injury. TNF- $\alpha$ is a potent inducer of IL-6 by various cells. Therefore, the association of CRP concentration with $H$. pylori infection could be explained by the actions of cytokines, hormones, or both.

In conclusion, the present cross-sectional study based on the three different subjects showed a significant association between $H$. pylori infection and serum hsCRP levels, supporting that $H$. pylori infection may increase the serum CRP. The possible attribution of $H$. pylori infection to CRP elevation could be a route to $H$. pylori negative subjects related coronary heart disease with the base of atherosclerosis. Since many factors influence the CRP levels, larger studies with information on potential confounding factors are necessary to confirm the association.

\section{Acknowledgments}

The authors are grateful to Ms. Yoko Mitsuda for their technical assistance. This work was supported in part by a Grant-in-Aid for Scientific Research on Spe- 
cial Priority Areas of Cancer from the Ministry of Education, Culture, Sports, Science and Technology of Japan.

\section{Conflict of Interest}

The authors have declared that no conflict of interest exists.

\section{References}

1. The EUROGAST Study Group. Epidemiology of, and risk factor for, Helicobacter pylori infection among 3194 asymptomatic subjects in 17 populations. Gut. 1993; 34: 1672-6.

2. Howden CW. Clinical expressions of Helicobacter pylori infection. Am J Med. 1996; 20: 27S-32S.

3. Roussos A, Philippou N, Gourgoulianis KI. Helicobacter pylori infection and respiratory diseases: a review. World J Gastroenterol. 2003; 9: 5-8.

4. Laurila A, Bloigu A, Nayha S, et al. Association of H. pylori infection with elevated serum lipids. Atherosclerosis. 1999; 142: 207-10.

5. Kinjo K, Sato H, Sato H. Prevalence of H. pylori infection and its link to coronary risk factors in Japanese patients with acute myocardial infarction. Circ J. 2002; 66: 805-10.

6. Niemela S, Karttunen T, Korhonen T, et al. Could H. pylori infection increase the risk of coronary heart disease by modifying serum lipid concentrations? Heart. 1996; 75: 573-5.

7. Cammaroat G, Pasceri V, Gasbarrini A, et al. Helicobacter pylori is an aetiologic factor for ischaemic heart disease: the case against. Digest Liver Dis. 2000; 32: 65-8.

8. Realdi G, Dore PM, Fastame L. Extradigestive manifestations of H. pylori infection. Fact and fiction. Dig Dis Sci. 1999; 44: 229-36.

9. Patel P, Gasbarrini G, Pretolani S, et al. Extradigestive diseases and H. pylori infection. Curr Opin Gastroenterol. 1997; 13: 52-5.

10. Le Moullec JM, Jullienne A, Chenais J, et al. The complete sequence of human preprocalcitonin. FEBS Lett. 1984; 167: 93-7.

11. Ridker PM, Cushman M, Stampfer MJ, et al. Inflammation, aspirin, and the risk of cardiovascular disease in apparently healthy men. N Engl J Med. 1997; 336: 973-9.

12. Ridker PM, Hennekens $\mathrm{CH}$, Buring JE, et al. C-reactive protein and other markers of inflammation in the prediction of cardiovascular disease in women. N Engl J Med. 2000; 342: 836-43.

13. Ridker PM, Rifai N, Rose L, et al. Comparison of C-reactive protein and low-density lipoprotein cholesterol levels in the prediction of first cardiovascular events. N Engl J Med. 2002; 347: 1557-65.

14. Paul MR, Julie EB, Nancy RC, et al. C-reactive protein, the metabolic syndrome and risk of incident cardiovascular events. Circulation. 2003; 107: 391-7.

15. Russell PT, Rozenn NL, Bruce MP, et al. Relationship of C-reactive protein to risk of cardiovascular disease in the elderly: results from the Cardiovascular Health Study and the Rural Health Promotion Project. Arterioscler Thromb Vasc Biol. 1997; 17: 1121-7.

16. John D, Peter W, Mary W, et al. Low grade inflammation and coronary heart disease: prospective study and updated meta-analysis. BMJ. 2000; 321: 199-204.

17. Wolfgang K, Malte S, Margit F, et al. C-reactive protein, a sensitive marker of inflammation predicts future risk of coronary heart disease in initially healthy middle-aged men: results from the MONICA (monitoring trends and determinants in cardiovascular disease) Augsburg Cohort Study, 1984 to 1992. Circulation. 1999; 99: 237-42.

18. Kuller LH, Tracy RP, Shaten J, et al. Relation of C-reactive protein and coronary artery heart disease in the MRFIT nested casecontrol study: multiple risk factor intervention trial. Am J Epidemiol. 1996; 144: 537-47.

19. Albert C, Ma J, Rifai N, et al. Prospective study of C-reactive protein, homocysteine, and plasma lipid levels as predictors of sudden cardiac death. Circulation. 2002; 105: 2595-9.

20. Pepys MB, Hirschfield GM. C-reactive protein: a critical update. J Clin Invest. 2003; 111:1805-12.

21. Brian FM. Evidence of smoke and atherosclerotic fire. CCJM. 2001; 68: 538-40.

22. Mendall MA, Goggin PM, Molineaux N, et al. Relation of Helicobacter pylori infection and coronary heart disease. Br Heart J. 1994; 71: 437-39.

23. Suat S, Bekir K, Mustafa A, et al. Do procalcitonin and C-reactive protein levels have a place in the diagnosis and follow-up of Helicobacter pylori infections? J Med Microbiol. 2004; 53: 639-44.

24. Mendall MA, Praful P, Lydia B, et al. C Reactive protein and its relation to cardiovascular risk factors: a population based cross sectional study. BMJ. 1996; 312: 1061-5.

25. Kalia N, Jones C, Bardhan KD, et al. Effects of genotypically different strains of Helicobacter pylori on human microvascular endothelial cells in vitro. Dig Dis Sci. 2001; 46: 54-61.

26. Sung JJY, Sanderson JE. Hyperhomocysteinanemia, Helicobacter pylori, and coronary heart disease. Heart. 1996; 76: 305-7.

27. Itou S, Goto Y, Kondo T, et al. Associations of methylenetetrahydrofolate reductase (MTHFR) polymorphism with hyperuricemia in Japanese. Int J Med Sci. 2007; 4: 98-104.

28. Nanri A, Moore MA, Kono S. Impact of C-reactive protein on disease risk and its relation to dietary factors: literature review. Asian Pac J Cancer Prev. 2007; 8: 167-77.

29. Ridker PM, Cannon CP, Morrow D, et al. Pravastatin or Atorvastatin Evaluation and Infection Therapy-Thrombolysis in Myocardial Infarction 22 (PROVE IT-TIMI 22) Investigators: C-reactive protein levels and outcomes after statin therapy. $\mathrm{N}$ Engl J Med. 2005; 352: 20-8.

30. Albert MA, Danielson E, Rifai N, et al. Effect of statin therapy on C-reactive protein levels: the pravastatin inflammation/CRP evaluation (PRINCE). a randomized trial and cohort study. JAMA. 2001; 286: 64-70.

31. Crabtree JE, Shallcross TM, Heatley RV, et al. Mucosal tumour necrosis factor alpha and interleukin-6 in patients with Helicobacter pylori associated gastritis. Gut. 1991; 32: 1473-7.

32. Moss SF, Legon S, Davies J, et al. Cytokine gene expression in Helicobacter pylori associated antral gastritis. Gut. 1994; 35: 1567-70.

33. Farsak B, Yildirir A, Akyon Y, et al. Detection of Chlamydia pneumoniae and Helicobacter pylori DNA in human atherosclerotic plaques by PCR. J Clin Microbiol. 2000; 38: 4408-11.

34. Ameriso SF, Fridman EA, Leiguarda RC, et al. Detection of Helicobacter pylori in human carotid atherosclerotic plaques. Stroke. 2001; 32: 385-91.

35. Cabana V, Siegel J, Sabesin S. Effects of the acute phase response on the concentration and density distribution of plasma lipids and apolipoproteins. J Lipid Res. 1989; 30: 39-49.

36. Gauldie J, Richards C, Northemann W, et al. IFNB2/BSF2/IL-6 is the monocyte-derived HSF that regulates receptor-specific acute phase gene regulation in hepatocytes. Ann NY Acad Sci. 1989; 557: 46-59.

37. Mackiewicz A, Speroff T, Ganapathim M, et al. Effects of cytokine combinations on acute phase protein production in two human hepatoma cell lines. J Immunol. 1991; 146: 3032-7.

38. Baumann H, Gauldie J. The acute phase response. Immunol Today. 1994; 15: 74-80. 